\title{
Predictores del consumo semanal de alcohol y sus consecuencias asociadas en universitarios consumidores intensivos de alcohol
}

\section{Predictors of weekly alcohol drinking and alcohol- related problems in binge-drinking undergraduates}

\author{
Patricia Motos Sellés*, María Teresa Cortés Tomás*, José Antonio Giménez Costa*, \\ Fernando Cadaveira Mahía** \\ * Departamento de Psicología Básica. Facultad de Psicología. Universitat de València \\ ** Departamento de Psicoloxia Clinica e Psicobioloxia. Facultad de Psicología. Universidade de Santiago de Compostela
}

\section{Resumen}

Las importantes implicaciones que genera el consumo intensivo de alcohol entre los jóvenes justifican el interés por determinar qué factores predicen su aparición. Concretamente, en este estudio se analiza el papel de la personalidad y edad de inicio en el consumo de alcohol en la predicción del consumo semanal de alcohol, y de todas estas variables en la predicción del número de consecuencias asociadas al consumo en jóvenes universitarios.

Se evalúan 213 consumidores intensivos de primer curso de la Universidad Complutense de Madrid. Todos ellos cumplimentaron un autoregistro de consumo, el BIS-11, el NEO-FFI y el IECI de consecuencias asociadas a la ingesta.

Los análisis de regresión de orden jerárquico muestran que la edad de inicio resulta ser una variable predictora relevante tanto en la explicación del consumo semanal como del número de consecuencias. Lo mismo puede decirse de la variable consumo semanal respecto a la del número de consecuencias.

En líneas generales, el influjo de las variables de personalidad es bastante limitado. Tan sólo mencionar la responsabilidad e impulsividad, que junto con la edad, llegan a explicar gran parte de la conducta de consumo semanal entre varones. En lo que respecta a las consecuencias derivadas del consumo, sólo resultan explicativas, aunque en menor medida que la edad y el consumo semanal, la impulsividad y el neuroticismo.

Esto justifica la necesidad de planificar intervenciones más ajustadas y de analizar nuevos predictores en el caso de las mujeres que permitan explicar en mayor medida su conducta de consumo semanal.

Palabras clave: consumo intensivo de alcohol, consecuencias, personalidad, edad inicio, universitarios.

\begin{abstract}
The important implications generated by binge drinking among university students justify the interest to determine which factors predict its occurrence. Specifically, this study aims to assess the role of personality and drinking onset in predicting weekly alcohol consumption, and the impact of the whole set of variables in predicting the number of consequences associated with consumption in undergraduates. Two hundred and thirteen freshmen who were intensive consumers (binge drinkers) from the University Complutense of Madrid were evaluated. All of them filled in a selfregistration of consumption, the BIS-11, the NEO-FFI and the IECI consequences associated with intake. The hierarchical regression analysis shows that the drinking onset appears to be a relevant predictor variable in explaining weekly consumption and the number of consequences. The same can be said of the weekly consumption variable with regard to the number of consequences. In general, the influence of personality is quite limited. It is interesting to point out that responsibility and impulsivity, along with age, explain most of the weekly consumption behavior among males. With respect to the consequences of consumption, only impulsivity and neuroticism contribute to explain them, but with less strength than age and weekly consumption. Our results justify the need to plan tighter interventions and consider new predictors that help to explain further weekly consumption in women.
\end{abstract}

Key words: binge drinking, consequences, personality, drinking onset, university students. 
$\mathrm{E}$ 1 Consumo Intensivo de Alcohol (CIA) -ingesta de 60 gramos o más en varones y 40 gramos o más en mujeres, durante un intervalo de 2-3 horas- (Hingson, Assailly y Williams, 2004; MSC, 2008; NIAAA, 2004) es una práctica generalizada entre los jóvenes (Anderson y Baumberg, 2006), ampliamente representada en población universitaria (Arata, Stafford y Tims, 2003; Calafat, 2007; Danielsson, Wennberg, Tengström y Romelsjö, 2010; March et al., 2010; Parada et al., 2011). Pese a que cada vez es más homogéneo el número de varones y mujeres que realizan esta ingesta, los primeros siguen mostrando mayores índices en cantidad y frecuencia de consumo (Cortés, Giménez, Motos y Cadaveira, 2014; Gliksman, Adlaf, Demers y Newton, 2003; O’Malley y Johnston, 2002; Wechsler, Dowda11, Davenport y Castillo, 1995).

Este patrón de consumo se asocia a múltiples problemas biopsicosociales (Martens et al., 2005; Neighbors, Walker y Larimer, 2003; Park, 2004; Ray, Turrisi, Abar y Peters, 2009), entre los que destacan los síntomas de intoxicación, problemas académicos o profesionales, problemas interpersonales, verse envuelto en conductas sexuales sin protección o no planificadas, conducir bajo los efectos del alcohol, meterse en peleas, sufrir lesiones, tener problemas legales o incluso causar daños a terceros (Cortés, 2010; Devos-Comby y Lange, 2008; Hingson, Zha y Weitzman, 2009; Kahler, Strong y Read, 2005; Mallett et al., 2011; Shield, Gmel, Patra y Rehm, 2012; Wechsler y Nelson, 2010).

La diversidad e importancia de algunas de estas consecuencias evidencia la necesidad de identificar con mayor precisión a los jóvenes que se encuentran en esta situación de riesgo, lo que permitirá fundamentar intervenciones ajustadas a sus necesidades (Cortés et al., 2014; Ibáñez, Ruipérez, Villa, Moya y Ortet, 2008).

Uno de los aspectos que en los últimos años ha generado interés es la relación entre variables de personalidad en consumidores de alcohol de diferente intensidad y frecuencia (Adan, 2012; Ibáñez et al., 2008; Woicik, Stewart, Pihl y Conrod, 2009) y la aparición de problemas biopsicosociales asociados (Cooper, Agocha y Sheldon, 2000; Ibáñez et al., 2008; Magid, MacLean y Colder, 2007; Mezquita, Stewart y Ruipérez, 2010; Ruipérez, Ibáñez, Villa y Ortet, 2006; Sher, Grekin y Williams, 2005).

Concretamente, se ha demostrado que rasgos incluidos en el modelo de los Cinco Factores de la Personalidad (Costa y McCrae, 1992), se vinculan con problemas asociados al consumo, tanto en población clínica de dependientes (Hopwood et al., 2007; Sher, Trull, Bartholow y Veith, 1999), como en abusadores de alcohol (Littlefield, Sher y Wood, 2010; Flory, Lynam, Milich, Leukefeld y Clayton, 2002) y CIA (Martin, 2011; Ruiz, Pincus y Dickinson, 2003).

Entre los factores más analizados en los diferentes tipos de consumidores están el Neuroticismo y la Extraversión (Martin, 2011; Mezquita et al., 2010; Read y O'Connor, 2006; Ruiz et al., 2003). Concretamente, en los CIAs, altos niveles de Neuroticismo correlacionan con un mayor número de problemas por consumo de alcohol (Conrod, Stewart, Comeau y Maclean, 2006; Cooper, et al., 2000), aportando un incremento significativo a la explicación de la varianza de estos problemas (Cooper et al., 2000; Ruiz et al., 2003).

Por su parte, la Extraversión se muestra directamente relacionada con variables relativas al patrón de consumo -cantidad de alcohol ingerido, frecuencia- y aporta un peso significativo en la predicción del CIA (Hussong, 2003; Martin, 2011; Ruiz et al., 2003).

En otros rasgos del modelo, como la Amabilidad, la investigación precedente señala que una puntuación baja suele asociarse tanto con un incremento de la cantidad de alcohol ingerida entre los CIAs (Kubicka, Matjcek, Dytrych y Roth, 2001; Ibáñez et al., 2010; Mestre, Viñas, Dutil y Moya, 2004; Ruiz et al., 2003) como de los problemas derivados de este consumo en jóvenes universitarios (Ruiz et al., 2003). No obstante, algunos trabajos muestran resultados contradictorios sobre la influencia de la Amabilidad en la ingesta realizada por consumidores intensivos (Hussong, 2003).

El rasgo Responsabilidad muestra un comportamiento similar al de Amabilidad, ya que la investigación también evidencia una asociación entre bajas puntuaciones en esta dimensión y un incremento de la ingesta entre CIAs (Ibáñez et al., 2010; Ruiz et al., 2003) y la aparición de problemas derivados de este consumo (Ruiz et al., 2003).

Por otra parte, en CIAs el rasgo de Apertura no aparece como un predictor significativo ni de la cantidad de alcohol ingerida, ni de los problemas generados a partir de la misma (Hussong, 2003; Ibáñez et al., 2010; Ruiz et al., 2003; Stewart, Loughlin y Rhyno, 2001).

Así, el perfil de personalidad característico de los jóvenes CIA podría definirse por niveles elevados en Neuroticismo (Conrod et al., 2006; Cooper et al., 2000; Hussong, 2003; Littlefield, Sher y Wood, 2009; Martín, 2011; Ruiz et al., 2003; Stewart et al., 2001) y Extraversión (Cooper et al., 2000; Hussong, 2003; Martin, 2011; Littlefield et al., 2009; Ruiz et al., 2003) y bajos en Responsabilidad (Ibáñez et al., 2010; Ruiz et al., 2003; Stewart et al., 2001) y Amabilidad (Hussong, 2003; Ibáñez et al., 2010; Ruiz et al., 2003; Stewart et al., 2001).

Uno de los instrumentos de personalidad que más se ha utilizado en la investigación relacionada con el consumo de alcohol, incluido el CIA, es el Inventario de Personalidad NEO Reducido de Cinco Factores (NEO-FFI, Costa y McCrae, 1992, 1999) que evalúa los rasgos de personalidad del Modelo de los Cinco Factores (Neuroticismo, Extraversión, Apertura, Amabilidad y Responsabilidad) (Boyle, Matthews y Saklofske, 2008; Hussong, 2003; Mezquita et al., 2010; Ruiz et al., 2003; Stewart et al., 2001).

Sin embargo, muchos investigadores advierten de la necesidad de complementar esta evaluación con un instrumento que dé cuenta de la multidimensionalidad del rasgo Impulsividad (Cyders, Flory, Rainer y Smith, 2009; Henges 
y Marczinski, 2012; Magid et al., 2007; Meda et al., 2009; Quilty y Oakman, 2004; Stanford et al., 2009; Whiteside y Lynam, 2001).

La literatura ha demostrado la existencia de una relación significativa entre la impulsividad y el CIA (Adan, 2012; Field, Schoenmakers y Wiers, 2008; Goudriaan, Grekin, Sher, 2011; MacKillop, Mattson, Anderson, Castelda y Donovick, 2007; Prado, Crespo, Brenlla y Páramo, 2007; Simons, Carey y Gaher, 2004; White et al., 2011) y entre este rasgo y la experimentación de consecuencias negativas como resultado de esta ingesta (Fischer y Smith, 2008; Littlefield et al., 2009; Ruiz et al., 2003; Simons, Gaher, Correia, Hunsen y Christopher, 2005; Simons et al., 2004).

Por todo ello, junto al NEO-FFI, se recomienda aplicar la Escala de Impulsividad de Barrat (BIS-11, Patton, Stanford y Barratt, 1995), al ser un instrumento utilizado en este tipo de consumidores (Balodis, Potenza y Olmstead, 2009; Carlson y Johnson, 2012).

Además, no puede obviarse en la relación entre personalidad y alcohol el papel del género. Algunos estudios apuntan que los rasgos propios del Neuroticismo son más característicos entre las mujeres consumidoras de diferentes intensidades, incluidas las CIAs, que tienden a experimentar un mayor número de problemas relacionados con el alcohol (Locke y Newcomb, 2001; Martin, Lynch, Pollock y Clark, 2000; Schuckit, Tipp, Bergman y Reich, 1997; Schutte, Hearst y Moos, 1997). Por otra parte, rasgos pertinentes a la Impulsividad parecen más relevantes entre los varones consumidores, incluidos los CIAs (Adan, 2012; Cortés et al., 2014; Fischer y Smith, 2008; Waldeck y Miller, 1997; Zuckermann y Kuhlman, 2000).

Sin embargo, muchos estudios sugieren que no existen diferencias estadísticamente significativas entre hombres y mujeres CIA en Neuroticismo (Ruiz et al., 2003) e Impulsividad (Balodis et al., 2009; Magid et al., 2007; Ruiz et al., 2003; Simons et al., 2004). Estos resultados señalan la necesidad de seguir evaluando el papel del género en la relación entre diferentes aspectos de la personalidad y el consumo de alcohol.

Finalmente, otra variable adicional a considerar por su vinculación con la aparición de consumos problemáticos, incluido el CIA, es la edad en la que se inicia la ingesta (Hingson, Heeren y Winter, 2006; Jenkins et al., 2011; Lo, 1996; Pitkänen, Lyyra y Pulkkinen, 2005; Warner y White, 2003; Warner, White y Johnson, 2007). Al respecto, se encuentran tanto estudios que demuestran esta relación (Bonomo, Bowes, Coffey, Carlin y Patton, 2004; DeWit, Adlaf, Offord y Ogborne, 2000; Grant, Stinson y Harford, 2001; Hingson y Zha, 2009; Livingston, Laslett y Dietze, 2008; Muthen y Muthen, 2000; Pilatti, Caneto, Garimaldi, Del Valle y Pautassi, 2013), como otros que la ponen en entredicho, vinculándola con otras variables, entre las que se incluyen aspectos de la personalidad (Afitska, Plant, Weir, Miller y Plant, 2008; Dawson, Goldstein, Chou, Ruan y Grant, 2008; Harford, 2003; Rossow y Kuntsche, 2013). Esto ha motivado que actualmente se continúe investigando el influjo de la edad de inicio como posible factor que contribuye al incremento en la probabilidad de aparición de consecuencias.

La presente investigación tiene como objetivos analizar en primer lugar, el peso predictivo de la edad de inicio y los rasgos de personalidad sobre la cantidad de alcohol que ingieren semanalmente jóvenes universitarios CIA. En segundo lugar, se verá el peso predictor de estas mismas variables -edad y rasgos de personalidad- junto con el consumo semanal de alcohol sobre el número de consecuencias experimentadas. En todo momento se considerará la perspectiva de género.

\section{Método}

\section{Participantes y procedimiento}

Para la selección de la muestra se realizó un muestreo estratificado de la población de estudiantes de primer curso de la Universidad Complutense de Madrid (UCM) durante el curso académico 2011/2012, a partir de los datos facilitados por el rectorado de dicha universidad. Se seleccionaron aquellas titulaciones de cada área de conocimiento (Ciencias Básicas, Ciencias Sociales, Ciencias de la Salud, Humanidades y Ciencias de la Educación) en función del número de matriculados, campus de ubicación de la titulación y distribución por sexos. En todos los casos se incluyó, al menos, un grupo de mañana y otro de tarde. Los cuestionarios se cumplimentaron en las aulas docentes y en horario lectivo (mañana o tarde), contando en todos los casos con miembros del equipo de investigación. La participación en el estudio era voluntaria, y se solicitaba un número de contacto para citarlos en la siguiente fase. Del total de estudiantes encuestados se seleccionaron 440 sujetos sin historia de trastornos psicopatológicos y/o neurológicos; abuso o dependencia de sustancias (incluido el alcohol), ni historia familiar de alcoholismo de primer grado. De ellos la mitad eran consumidores intensivos de alcohol y el resto abstemios o consumidores de una cantidad reducida, muy por debajo de lo que se considera un CIA. De todos ellos, tras firmar un consentimiento informado, se registró entre los múltiples aspectos evaluados el patrón de consumo, así como sus determinantes cognitivos y de personalidad. Los datos aquí presentados forman parte de un estudio longitudinal de cohortes al incluir también evaluación neuropsicológica, si bien, en este trabajo al centrarse solamente en datos ofrecidos en la primera fase de la investigación puede decirse que se maneja información de un estudio analítico-transversal. Concretamente, se evalúan los resultados obtenidos por los 213 estudiantes que realizan CIA (MSC, 2008). Un 56.8\% de la muestra son mujeres $(n=121)$. La edad media es de 18.20 años $(S D=.414)$.

\section{Variables}

Edad de inicio. Se indica la edad en la que se inicia el consumo de alcohol por decisión propia. 
Patrón de consumo. Se registra el número de veces que se ingiere alcohol durante los últimos seis meses. En una tabla elaborada ad hoc se anota el número de consumiciones ingeridas cada día de una semana de consumo habitual durante esos seis meses, siguiendo un procedimiento similar a Neighbors, Lee, Lewis, Fossos y Larimer (2007). También se registra el tipo de alcohol consumido y la hora en la que se realiza cada ingesta. Toda esta información permite, tomando como referencia las Unidades de Bebida Estándar en España (Rodríguez-Martos, Gual y Llopis, 1999), calcular los gramos de alcohol ingeridos en cada consumición. A partir de los mismos se genera diferentes variables: suma de gramos de alcohol ingeridos a la semana (resultado de sumar los gramos de alcohol que se ingieren cada día de la semana, extraídos del autorregistro de consumo) y tipo de consumo (CIA-NoCIA). Para obtener esta última variable se calcula los gramos máximos ingeridos durante el intervalo de 2/3 horas de mayor consumo, etiquetando como consumidor intensivo de alcohol a aquellos chicos que alcanzan 60 o más gramos de alcohol y a aquellas chicas que alcanzan 40 o más gramos (MSC, 2008).

Rasgos de personalidad. Se utiliza la versión española (Cordero, Pamos y Seisdedos, 2008) del Inventario de Personalidad Reducido NEO-FFI de Costa y McCrae (1999). Consta de 60 ítems que evalúan el Modelo de Cinco Factores de la personalidad (Neuroticismo, Extraversión, Apertura, Amabilidad y Responsabilidad), mediante una escala de respuesta Likert de 5 puntos que va desde 0 (totalmente en desacuerdo) a 4 (muy de acuerdo). En este estudio, se han utilizado las puntuaciones $\mathrm{T}$ de las 5 escalas (el rango de dichas puntuaciones varía entre 25 y 75; se considera una puntuación alta a partir de 55 y muy alta a partir de 65). Este instrumento obtiene una buena consistencia interna en multitud de estudios con población universitaria CIA - alfa de Cronbach entre 0.71 y 0.85- (Mezquita et al., 2010; Ruiz et al., 2003; Sanz, Silva y Avia, 1999).

La Impulsividad se mide mediante el instrumento BIS11 de Barratt (Patton et al., 1995; adaptación española de Oquendo et al., 2001), compuesto por 30 ítems tipo Likert de 4 puntos: 0 (nunca/raramente), 1 (ocasionalmente), 3 (a menudo) y 4 (casi siempre) que miden Impulsividad Cognitiva, Motora y No planificada. La suma de las puntuaciones de estas tres últimas constituye la escala de Impulsividad Total utilizada en este estudio (con un rango de puntuaciones que va de 0 a 120). Este instrumento ha mostrado buena consistencia interna en población estudiantil consumidora -alfa de Cronbach entre 0.79 y 0.83- (Hair y Hampson, 2006; Patton et al., 1995; Stanford et al., 2009).

Consecuencias. Se evalúa con el apartado correspondiente del instrumento IECI (Cortés et al., 2012). La escala incluye 30 ítems que hacen referencia a diferentes consecuencias que cada joven afirma estar experimentando debido a su consumo. Éstas se refieren a síntomas físicos (he tenido resaca, mareos y vómitos; etc.); pérdida del control (he bebido más de lo planeado; etc.); conductas de riesgo (he realizado prácticas sexuales de riesgo; etc.); dependencia física (necesito mayor cantidad de alcohol que hace unos años; etc.); autopercepción (consumir alcohol me hace sentir culpable, etc.); consecuencias académicas o profesionales (he descuidado mis responsabilidades a causa de la bebida; etc.); consecuencias socio-interpersonales (cuando bebo digo cosas de las que luego me arrepiento; etc.) y otras consecuencias (tener problemas económicos a causa de la bebida; etc.). Todos ellos se responden mediante una escala dicotómica (Sí/No). Esta escala ha mostrado buen ajuste en estudios previos, alcanzando un alfa de Cronbach superior a .807 (Cortés et al., 2012; Motos, 2013). En el presente estudio se utiliza la suma de todas las consecuencias que cada joven señalaba haber presentado en los últimos 6 meses, constituyendo la variable número de consecuencias (con un rango de puntuaciones entre 0 y 30 ).

\section{Análisis de datos}

Mediante el paquete estadístico IBM SPSS Statistics 19 se llevaron a cabo análisis descriptivos de las variables consumo intensivo, consumo semanal, edad de inicio, rasgos de personalidad y número de consecuencias para la muestra general y por sexos. Además, para comprobar la posible existencia de diferencias en función del género se efectuaron contrastes de medias (mediante la prueba $t$ de Student) en estas mismas variables.

A continuación se examinaron las correlaciones de orden cero (mediante el coeficiente de correlación de Pearson) entre número de consecuencias, consumo semanal, edad de inicio, sexo, cinco rasgos de personalidad del NEO-FFI e Impulsividad Total. Esto permitió confirmar qué elementos estaban más fuertemente asociados con el consumo y sus consecuencias e identificar variables que presentasen relaciones bivariadas imprevistas.

Como paso previo a los análisis de regresión, se aplicó la transformación de Blom (1958) a todas las variables continuas utilizadas en los mismos (excepto NEO-FFI), con el fin de evitar los sesgos en las frecuencias de las medidas y de mantener la coherencia de los datos. Mediante este método los casos son ordenados por rangos, el rango de cada caso se convierte en un percentil y finalmente se normalizan las medidas. El resultado es una puntuación $z$ de rangos, que reduce al mínimo el impacto espurio de los casos extremos.

Finalmente, se llevaron a cabo tres análisis de regresión de orden jerárquico (uno para el total de la muestra y uno para cada sexo) para detectar las contribuciones únicas de las variables edad de inicio, sexo, Neuroticismo, Extraversión, Apertura, Amabilidad, Responsabilidad e Impulsividad Total sobre la cantidad de alcohol ingerido a la semana. Estas variables se introdujeron en ocho pasos separados.

Por otra parte, para predecir el porcentaje de varianza sobre las consecuencias, se llevó a cabo otro análisis de regresión de orden jerárquico. En este caso, para el total de la muestra, debido a que no se observaron diferencias por gé- 
nero en las pruebas $t$ ni en las correlaciones. Concretamente, se introdujeron las variables edad de inicio, gramos semanales, Neuroticismo, Extraversión, Apertura, Amabilidad, Responsabilidad e Impulsividad Total en ocho pasos separados.

\section{Resultados}

La Tabla 1 muestra como tanto los varones (121.63g) como las mujeres $(89.09 \mathrm{~g})$ ingieren el doble de gramos de alcohol que definen un CIA (60g- $40 \mathrm{~g}$ respectivamente). Además, como se aprecia en la diferencia entre este consumo y el reflejado por la variable Gramos semanales de alcohol, complementan este consumo con otros de menor cuantía a lo largo de la semana. Al considerar el consumo total semanal, un tercio de las mujeres y una cuarta parte de los varones superan el límite fijado por la WHO (2007) para definir el consumo de riesgo semanal (280g en varones y $170 \mathrm{~g}$ en mujeres).
Reparando en las diferencias de género, la misma tabla muestra como los varones consumen significativamente mayor cantidad de alcohol a la semana (209.24g vs. 152.77g; $\mathrm{t}=-4.042 ; p<.000)$ y durante una sesión CIA que las mujeres (121.63g vs. 89.09g; t=-4.988; $p<.000)$. En cambio, no se aprecian diferencias significativas en la edad de inicio en el consumo, ni en el número de consecuencias experimentadas.

Si se atiende a los rasgos de personalidad, son los varones los que obtienen una media significativamente superior en Amabilidad (58.82 vs. 53.23; $\mathrm{t}=-6.209 ; p<.000)$ e Impulsividad ( 47.43 vs. $43.64 ; \mathrm{t}=-2.201 ; p<.029$ ). En general, las puntuaciones de las escalas de personalidad pueden calificarse de altas, destacando sobre el resto la de Neuroticismo, cuyo perfil se define como muy alto, según el baremo del NEOFFI. No se observan diferencias de género en Neuroticismo, Apertura, Extraversión ni en Responsabilidad.

Tabla 1.

Variables de consumo, número de consecuencias y rasgos de personalidad, diferenciadas por sexos.

\begin{tabular}{|c|c|c|c|c|c|c|c|c|}
\hline \multirow{2}{*}{$\begin{array}{l}\text { Ítem } \\
\text { Edad de inicio }\end{array}$} & \multirow{2}{*}{$\begin{array}{c}\text { Media } \\
14.94\end{array}$} & \multirow{2}{*}{$\frac{\text { Sd }}{1.25}$} & \multicolumn{2}{|c|}{ Varones (M, sd) } & \multicolumn{2}{|c|}{ Mujeres (M, sd) } & \multirow{2}{*}{$\frac{\mathbf{t}}{-1.538}$} & \multirow{2}{*}{$\begin{array}{r}\mathbf{p} \\
.126\end{array}$} \\
\hline & & & 15.10 & 1.37 & 14.83 & 1.13 & & \\
\hline Gramos semanales de alcohol & 177.16 & 99.89 & 209.24 & 115.14 & 152.77 & 78.65 & -4.042 & .000 \\
\hline Gramos en una sesión $\mathrm{CI}^{1}$ & 103.15 & 47.37 & 121.63 & 54.35 & 89.09 & 35.54 & -4.988 & .000 \\
\hline $\begin{array}{l}\text { Número de consecuencias } \\
\text { Rasgos de personalidad }\end{array}$ & 7.79 & 3.53 & 8.22 & 3.58 & 7.47 & 3.46 & -1.534 & .126 \\
\hline Neuroticismo & 69.77 & 4.48 & 70.30 & 3.69 & 69.38 & 4.97 & -1.540 & .125 \\
\hline Extraversión & 58.95 & 5.35 & 59.28 & 4.96 & 58.70 & 5.64 & -0.783 & .435 \\
\hline Apertura & 62.22 & 6.51 & 62.62 & 6.22 & 61.92 & 6.74 & -0.778 & .437 \\
\hline Amabilidad & 55.63 & 7.04 & 58.82 & 6.32 & 53.23 & 6.61 & -6.209 & .000 \\
\hline Responsabilidad & 58.44 & 6.60 & 57.98 & 6.83 & 58.79 & 6.42 & 0.878 & .381 \\
\hline Impulsividad Total & 45.28 & 12.58 & 47.43 & 11.83 & 43.64 & 12.94 & -2.201 & .029 \\
\hline
\end{tabular}

Note: ${ }^{1} \mathrm{CIA}$ : Consumo Intensivo de Alcohol

En la primera columna de la Tabla 2 se muestran las correlaciones de la cantidad de alcohol ingerido a la semana con la edad de inicio en el consumo, el sexo y los rasgos de personalidad del NEO-FFI y del BIS-11, para la muestra general.

La edad de inicio muestra una correlación negativa sobre la cantidad de alcohol ingerido en una semana. En cambio, el sexo correlaciona de manera positiva con el consumo. De ahí, que se hayan efectuado análisis de regresión jerárquica diferenciados por género.

En la segunda columna de la Tabla 2 se muestran las correlaciones de las consecuencias experimentadas con la edad de inicio en el consumo, el sexo, los gramos semanales ingeridos y los rasgos de personalidad del NEO-FFI y del BIS-11, para la muestra general.

El consumo semanal correlaciona positiva y significativamente con el número de consecuencias experimentadas. Por el contrario, la edad de inicio muestra una relación negativa, igual que en el caso anterior. Por su parte, la Impul- sividad correlaciona más fuertemente con las consecuencias derivadas del consumo.

En este caso no aparecen diferencias en función del género, por lo que no se han realizado posteriores análisis de regresión jerárquica separados.

Tabla 2.

Correlaciones de variables de consumo y rasgos de personalidad con el consumo semanal y número de consecuencias.

\begin{tabular}{lcc}
\hline & Gramos semanales & $\mathbf{N}^{0}$ consecuencias \\
\hline Edad de inicio & $-.267^{* *}$ & $-.288^{* *}$ \\
Sexo & $.281^{* *}$ & .091 \\
Gramos semanales & - & $.332^{* *}$ \\
$\quad$ Rasgos de personalidad & & $.154^{*}$ \\
Neuroticismo & -.040 & $.135^{*}$ \\
Extraversion & .078 & .008 \\
Apertura & $.120^{*}$ & $.152^{*}$ \\
Amabilidad & $.180^{* *}$ & -.061 \\
Responsabilidad & -.111 & $.310^{* *}$ \\
Impulsividad Total & $.185^{* *}$ & \\
\hline
\end{tabular}


Las Tablas 3, 4 y 5 presentan los resultados de los análisis de regresión de orden jerárquico para predecir la cantidad de alcohol ingerido a la semana, tanto para la muestra global como para cada uno de los sexos.

Únicamente las variables edad de inicio y sexo resultan significativas para la muestra total, explicando un $4.8 \%$ y un $9.6 \%$ de la varianza, respectivamente.

En el caso de la primera variable, la contribución de la varianza también es significativa en ambos sexos, siendo superior en los varones (10.6\%). Además, la Responsabilidad y la Impulsividad, resultan significativas para los varones. La cantidad de varianza aportada a la predicción del consumo semanal es de $9.4 \%$ y de $6.9 \%$, respectivamente. Entre las mujeres, únicamente destaca la edad de inicio con un $3.8 \%$ de varianza explicada.

El mayor porcentaje de varianza explicada del consumo semanal lo obtienen los varones (30.4\%). Este porcentaje es once puntos superior al de la muestra total $(19.4 \%)$ y diez puntos superior cuando se compara con el de las mujeres $(9.5 \%)$.

Tabla 3.

Análisis de regresión de orden jerárquico para predecir la cantidad de alcohol ingerido semanal, en la muestra total.

\begin{tabular}{|c|c|c|c|c|c|c|c|c|c|c|c|c|c|c|c|c|c|c|c|c|c|}
\hline & \multirow[b]{2}{*}{$\mathbf{R}$} & \multirow[b]{2}{*}{$\mathbf{R}^{2}$} & \multirow[b]{2}{*}{$\begin{array}{c}\text { Error } \\
\text { estimación }\end{array}$} & \multirow[b]{2}{*}{$\Delta \mathbf{R}^{2}$} & \multirow[b]{2}{*}{$\mathrm{F} \Delta \mathbf{R}^{2}$} & \multicolumn{2}{|c|}{ Paso 1} & \multicolumn{2}{|c|}{ Paso 2} & \multicolumn{2}{|c|}{ Paso 3} & \multicolumn{2}{|c|}{ Paso 4} & \multicolumn{2}{|c|}{ Paso 5} & \multicolumn{2}{|c|}{ Paso 6} & \multicolumn{2}{|c|}{ Paso 7} & \multicolumn{2}{|c|}{ Paso 8} \\
\hline & & & & & & $\beta$ & $\begin{array}{l}\text { Valor } p \\
\text { para } \beta\end{array}$ & $\beta$ & $\begin{array}{l}\text { Valor } \mathbf{p} \\
\text { para } \beta\end{array}$ & $\beta$ & $\begin{array}{c}\text { Valor } \mathbf{p} \\
\text { para } \beta\end{array}$ & $\beta$ & $\begin{array}{c}\text { Valor } p \\
\text { para } \beta\end{array}$ & $\beta$ & $\begin{array}{l}\text { Valor } p \\
\text { para } \beta\end{array}$ & $\beta$ & $\begin{array}{l}\text { Valor } p \\
\text { para } \beta\end{array}$ & $\beta$ & $\begin{array}{l}\text { Valor } \mathbf{p} \\
\text { para } \beta\end{array}$ & $\beta$ & $\begin{array}{r}\text { Valor } \mathbf{p} \\
\text { para } \beta\end{array}$ \\
\hline 1 & .218 & .048 & .798 & .048 & $10.266^{* *}$ & -.218 & $-3.204^{* *}$ & & & & & & & & & & & & & & \\
\hline 2 & .380 & .144 & .759 & .096 & $22.968^{* * *}$ & -.254 & $-3.895^{* * *}$ & .312 & $4.793^{* * *}$ & & & & & & & & & & & & \\
\hline 3 & .394 & .155 & .756 & .011 & 2.608 & -.239 & $-3.643^{* * *}$ & .295 & $4.480^{* * *}$ & .106 & 1.615 & & & & & & & & & & \\
\hline 4 & .402 & .161 & .755 & .006 & 1.555 & -.246 & $-3.738^{* * *}$ & .262 & $3.709^{* * *}$ & .097 & 1.465 & .088 & 1.247 & & & & & & & & \\
\hline 5 & .412 & .170 & .753 & .009 & 2.063 & -.253 & $-3.844^{* * *}$ & .269 & $3.801^{* * *}$ & .084 & 1.266 & .066 & .909 & .096 & 1.436 & & & & & & \\
\hline 6 & .421 & .177 & .751 & .008 & 1.843 & -.252 & $-3.848^{* * *}$ & .262 & $3.704^{* * *}$ & .073 & 1.085 & .073 & 1.010 & .100 & 1.501 & -.08 & -1.35 & & & & \\
\hline 7 & .427 & .182 & .751 & .005 & 1.132 & -.259 & $-3.932^{* * *}$ & .262 & $3.700^{* * *}$ & .060 & .877 & .070 & .963 & .090 & 1.341 & -.11 & -1.564 & .073 & 1.064 & & \\
\hline 8 & .441 & .194 & .747 & .012 & 3.002 & -.262 & $-3.990^{* * *}$ & .264 & $3.754^{* * *}$ & .069 & 1.011 & .088 & 1.212 & .093 & 1.379 & -.09 & -1.460 & .096 & 1.386 & -.12 & -1.733 \\
\hline
\end{tabular}

Note: Paso 1. Edad de inicio; Paso 2.Sexo; Paso 3. Impulsividad Total; Paso 4. Amabilidad; Paso 5. Apertura; Paso 6. Responsabilidad; Paso 7. Extraversión

Paso 8. Neuroticismo.

$* .01<p \leq .05 \quad * * .001<p \leq .01 \quad * * * p \leq .001$

Tabla 4.

Análisis de regresión de orden jerárquico en varones para predecir la cantidad de alcohol ingerido semanal.

\begin{tabular}{|c|c|c|c|c|c|c|c|c|c|c|c|c|c|c|c|c|c|c|c|}
\hline & & & & & & \multicolumn{2}{|c|}{ Paso 1} & \multicolumn{2}{|c|}{ Paso 2} & \multicolumn{2}{|c|}{ Paso 3} & \multicolumn{2}{|c|}{ Paso 4} & \multicolumn{2}{|c|}{ Paso 5} & \multicolumn{2}{|r|}{ Paso 6} & \multicolumn{2}{|c|}{ Paso 7} \\
\hline & $\mathbf{R}$ & $\mathbf{R}^{2}$ & $\begin{array}{c}\text { Error } \\
\text { estimación }\end{array}$ & $\Delta \mathbf{R}^{2}$ & $\mathbf{F} \Delta \mathbf{R}^{2}$ & $\beta$ & $\begin{array}{l}\text { Valor } \mathbf{p} \\
\text { para } \beta\end{array}$ & $\beta$ & $\begin{array}{l}\text { Valor } \mathbf{p} \\
\text { para } \beta\end{array}$ & $\beta$ & $\begin{array}{l}\text { Valor } \mathbf{p} \\
\text { para } \beta\end{array}$ & $\beta$ & $\begin{array}{l}\text { Valor } \mathbf{p} \\
\text { para } \beta\end{array}$ & $\beta$ & $\begin{array}{l}\text { Valor } \mathbf{p} \\
\text { para } \beta\end{array}$ & $\beta$ & $\begin{array}{c}\text { Valor } p \\
\text { para } \beta\end{array}$ & $\beta$ & $\begin{array}{c}\text { Valor } \mathbf{p} \\
\text { para } \beta\end{array}$ \\
\hline 1 & .326 & .106 & .8137 & .106 & $10.204^{* *}$ & -.326 & $-3.194^{* *}$ & & & & & & & & & & & & \\
\hline 2 & .448 & .200 & .7741 & .094 & $10.014^{* *}$ & -.302 & $-3.106^{* *}$ & -.308 & $-3.164 * *$ & & & & & & & & & & \\
\hline 3 & .519 & .270 & .7442 & .069 & $7.976^{*}$ & -.220 & $-2.241^{*}$ & -.313 & $-3.347^{* *}$ & .276 & $2.824^{* *}$ & & & & & & & & \\
\hline 4 & .535 & .286 & .7403 & .016 & 1.897 & -.225 & $-2.306^{*}$ & -.296 & $-3.153^{* *}$ & .298 & $3.024^{* *}$ & -.131 & -1.377 & & & & & & \\
\hline 5 & .548 & .301 & .7371 & .015 & 1.718 & -.235 & $-2.408^{*}$ & -.273 & $-2.878 * *$ & .296 & $3.020 * *$ & -.167 & -1.696 & .128 & 1.311 & & & & \\
\hline 6 & .549 & .302 & .7411 & .001 & .112 & -.236 & $-2.408^{*}$ & -.275 & $-2.878 * *$ & .295 & $2.985^{* *}$ & -.168 & -1.689 & .123 & 1.239 & .032 & .335 & & \\
\hline 7 & .551 & .304 & .7445 & .002 & .257 & -.239 & $-2.423^{*}$ & -.283 & $-2.909 * *$ & .289 & $2.895^{* *}$ & -.176 & -1.743 & .124 & 1.242 & .036 & .377 & .050 & .507 \\
\hline
\end{tabular}

Note: Paso 1. Edad de inicio; Paso 2. Responsabilidad; Paso 3. Impulsividad Total; Paso 4. Neuroticismo; Paso 5. Apertura; Paso 6. Amabilidad; Paso 7. Extraversión $* .01<p \leq .05 \quad * * .001<p \leq .01 \quad * * * \quad p \leq .001$

Tabla 5 .

Análisis de regresión de orden jerárquico en mujeres para predecir la cantidad de alcohol ingerido semanal.

\begin{tabular}{|c|c|c|c|c|c|c|c|c|c|c|c|c|c|c|c|c|c|c|c|}
\hline & & & & & & \multicolumn{2}{|c|}{ Paso 1} & \multicolumn{2}{|c|}{ Paso 2} & \multicolumn{2}{|c|}{ Paso 3} & \multicolumn{2}{|c|}{ Paso 4} & \multicolumn{2}{|c|}{ Paso 5} & \multicolumn{2}{|c|}{ Paso 6} & \multicolumn{2}{|r|}{ Paso 7} \\
\hline & $\mathbf{R}$ & $\mathbf{R}^{2}$ & $\begin{array}{c}\text { Error } \\
\text { estimación }\end{array}$ & $\Delta \mathbf{R}^{2}$ & $\mathbf{F} \Delta \mathbf{R}^{2}$ & $\beta$ & $\begin{array}{l}\text { Valor } \mathbf{p} \\
\text { para } \beta\end{array}$ & $\beta$ & $\begin{array}{l}\text { Valor } \mathbf{p} \\
\text { para } \beta\end{array}$ & $\beta$ & $\begin{array}{l}\text { Valor } \mathbf{p} \\
\text { para } \beta\end{array}$ & $\beta$ & $\begin{array}{l}\text { Valor } p \\
\text { para } \beta\end{array}$ & $\beta$ & $\begin{array}{l}\text { Valor } \mathbf{p} \\
\text { para } \beta\end{array}$ & $\beta$ & $\begin{array}{l}\text { Valor } \mathbf{p} \\
\text { para } \beta\end{array}$ & $\beta$ & $\begin{array}{l}\text { Valor } \mathbf{p} \\
\text { para } \beta\end{array}$ \\
\hline 1 & .195 & .038 & .7158 & .038 & $4.619 *$ & -.195 & $-2.149 *$ & & & & & & & & & & & & \\
\hline 2 & .248 & .062 & .7100 & .024 & 2.922 & -.217 & $-2.386^{*}$ & .155 & 1.709 & & & & & & & & & & \\
\hline 3 & .259 & .067 & .7109 & .006 & .701 & -.221 & $-2.428^{*}$ & .132 & 1.386 & .079 & .837 & & & & & & & & \\
\hline 4 & .274 & .075 & .7110 & .008 & .956 & -.220 & $-2.411^{*}$ & .158 & 1.597 & .074 & .776 & -.092 & -.978 & & & & & & \\
\hline 5 & .297 & .088 & .7092 & .013 & 1.594 & -.225 & $-2.467 *$ & .149 & 1.505 & .043 & .436 & -.119 & -1.239 & .123 & 1.262 & & & & \\
\hline 6 & .306 & .094 & .7101 & .006 & .699 & -.220 & $-2.410 *$ & .134 & 1.333 & .033 & .330 & -.122 & -1.274 & .122 & 1.252 & .078 & .836 & & \\
\hline 7 & .309 & .095 & .7126 & .002 & .228 & -.223 & $-2.430 *$ & .142 & 1.390 & .041 & .412 & -.120 & -1.239 & .125 & 1.280 & .065 & .661 & -.047 & -.478 \\
\hline
\end{tabular}

Note: Paso 1. Edad de inicio; Paso 2. Extraversión; Paso 3. Apertura; Paso 4. Neuroticismo; Paso 5. Amabilidad; Paso 6. Responsabilidad; Paso 7. Impulsividad Total $* .01<p \leq .05 \quad * * .001<p \leq .01 \quad * * * \quad p \leq .001$ 
La Tabla 6 presenta los resultados para la muestra global de los análisis de regresión de orden jerárquico para predecir las consecuencias derivadas del consumo de alcohol.

El modelo total aporta a la predicción de la varianza un $20.2 \%$. La variable que explica el mayor porcentaje de varianza es la edad de inicio $(6.9 \%)$. Le sigue el consumo semanal $(5.2 \%)$ y los rasgos de Impulsividad $(4.4 \%)$ y Neuroticismo $(1.8 \%)$.

Tabla 6.

Análisis de regresión de orden jerárquico para predecir el número de consecuencias derivadas del CIA, en la muestra total.

\begin{tabular}{|c|c|c|c|c|c|c|c|c|c|c|c|c|c|c|c|c|c|c|c|c|c|c|c|}
\hline & & & & & & \multicolumn{2}{|c|}{ Paso 1} & \multicolumn{2}{|c|}{ Paso 2} & \multicolumn{2}{|c|}{ Paso 3} & \multicolumn{2}{|c|}{ Paso 4} & \multicolumn{2}{|c|}{ Paso 5} & \multicolumn{2}{|c|}{ Paso 6} & \multicolumn{2}{|c|}{ Paso 7} & \multicolumn{2}{|c|}{ Paso 8} & \multicolumn{2}{|c|}{ Paso 9} \\
\hline & $\mathbf{R}$ & $\begin{array}{l}R^{2} \\
\text { e }\end{array}$ & $\begin{array}{l}\text { Error } \\
\text { timaci }\end{array}$ & $n^{\Delta R^{2}}$ & $\mathbf{F} \Delta \mathbf{R}^{2}$ & $\beta$ & $\begin{array}{l}\text { Valor } \mathbf{p} \\
\text { para } \beta\end{array}$ & $\beta$ & $\begin{array}{l}\text { Valor } p \\
\text { para } \beta\end{array}$ & $\beta$ & $\begin{array}{l}\text { Valor } \mathbf{p} \\
\text { para } \beta\end{array}$ & $\beta$ & $\begin{array}{l}\text { Valor } \mathbf{p} \\
\text { para } \beta\end{array}$ & $\beta$ & $\begin{array}{l}\text { Valor } \mathbf{p} \\
\text { para } \beta\end{array}$ & $\beta$ & $\begin{array}{l}\text { Valor } \mathbf{p} \\
\text { para } \beta\end{array}$ & $\beta$ & $\begin{array}{l}\text { Valor } \mathbf{p} \\
\text { para } \beta\end{array}$ & $\beta$ & $\begin{array}{l}\text { Valor } \mathbf{p} \\
\text { para } \beta\end{array}$ & $\beta$ & $\begin{array}{r}\text { Valor } \mathbf{p} \\
\text { para } \beta\end{array}$ \\
\hline 1 & .263 & .069 & .8744 & .069 & $15.177^{* * *}$ & -.263 & $-3.896^{* * *}$ & & & & & & & & & & & & & & & & \\
\hline 2 & .348 & .121 & .8517 & .052 & $12.048^{* *}$ & -.212 & $-3.145^{* *}$ & .234 & $3.471^{* *}$ & & & & & & & & & & & & & & \\
\hline 3 & .405 & .164 & .8324 & .044 & $10.567^{* *}$ & -.193 & $-2.925^{* *}$ & .199 & $2.994^{* *}$ & .213 & $3.251^{* *}$ & & & & & & & & & & & & \\
\hline 4 & .428 & .183 & .8252 & .018 & $4.565^{*}$ & -.195 & $-2.977^{* *}$ & .208 & $3.148^{* *}$ & .192 & $2.930^{* *}$ & .137 & $2.137^{*}$ & & & & & & & & & & \\
\hline 5 & .435 & .190 & .8239 & .007 & 1.658 & -.209 & $-3.153^{* *}$ & .190 & $2.824^{* *}$ & .183 & $2.776^{* *}$ & .120 & 1.828 & .087 & 1.288 & & & & & & & & \\
\hline 6 & .441 & .194 & .8234 & .005 & 1.230 & -.218 & $-3.265^{* *}$ & .184 & $2.725^{* *}$ & .173 & $2.604^{* *}$ & .103 & 1.532 & .084 & 1.253 & .074 & 1.109 & & & & & & \\
\hline 7 & .441 & .194 & .8255 & .000 & .003 & -.218 & $-3.225^{* *}$ & .183 & $2.611^{* *}$ & .173 & $2.585^{* *}$ & .103 & 1.524 & .083 & 1.165 & .074 & 1.108 & -.070 & -1.067 & & & & \\
\hline 8 & .443 & .197 & .8264 & .002 & .524 & -.220 & $-3.249^{* *}$ & .178 & $2.521^{*}$ & .165 & $2.436^{*}$ & .105 & 1.557 & .087 & 1.209 & .085 & 1.240 & .002 & .026 & -.05 & -.724 & & \\
\hline 9 & .449 & .202 & .8259 & .005 & 1.267 & -.214 & $-3.145^{* *}$ & .186 & $2.621^{* *}$ & .173 & $2.537^{*}$ & .108 & 1.590 & .102 & 1.401 & .095 & 1.367 & -.005 & -.068 & -.047 & $7-.699$ & -.076 & -1.126 \\
\hline
\end{tabular}

Note: Paso 1. Edad de inicio; Paso 2. Consumo semanal; Paso 3. Impulsividad Total; Paso 4. Neuroticismo; Paso 5. Amabilidad; Paso 6. Extraversión; Paso 7. Sexo; Paso 8. Responsabilidad; Paso 9. Apertura

$* .01<p \leq .05 \quad * * .001<p \leq .01 \quad * * * p \leq .001$

\section{Discusión}

Los datos de consumo analizados en esta muestra confirman la tendencia observada en trabajos anteriores (Cadaveira, 2010; Cortés, 2012; Cortés et al., 2014; White, Kraus y Swartzwelder, 2006), que indican la homogeneización de un patrón de consumo CIA entre los jóvenes que llega a doblar los gramos de alcohol requeridos para poder definirlo. Es importante resaltar que estos jóvenes CIA no suelen clasificarse como consumidores de riesgo semanal tal como los define la OMS (WHO, 2007), de ahí la dificultad de poderlos identificar si únicamente se atiende al consumo semanal (Cortés, Motos y Giménez, 2013).

Los jóvenes presentan puntuaciones elevadas en los cinco factores evaluados, pudiéndose destacar dos aspectos. Por un lado, se observa una relación entre todos ellos similar a la enunciada en la introducción (Conrod et al., 2006: Cooper et al., 2000; Hussong, 2003; Ibáñez et al., 2010; Littlefield et al., 2009; Martín, 2011; Ruiz et al., 2003; Stewart et al., 2001), ya que las puntuaciones más altas se encuentran en Neuroticismo y Extraversión, quedando en un segundo plano las de Amabilidad y Responsabilidad. Esto permite definir al CIA como una persona con reacciones emocionales que pueden interferir en su propio equilibrio, haciéndolo actuar de manera irracional e incluso rígida. Además, sería una persona sociable, amante de las fiestas, con necesidad de hablar con gente, pero al mismo tiempo impulsiva, despreocupada e incluso con cierta tendencia a ser agresiva en ocasiones. El altruismo, la sensibilidad con los demás, la autodisciplina y la eficiencia, aunque presentes, no estarían representando con la misma intensidad a estos jóvenes.
El otro aspecto a destacar es que a diferencia de otras investigaciones (Hussong, 2003; Ibáñez et al., 2010; Ruiz et al., 2003; Stewart et al., 2001), en este caso no se descarta de la evaluación la influencia del rasgo Apertura, al mostrarse tan relevante como los demás. Este resultado no sorprende si se tiene en cuenta que la muestra estudiada ha sido de universitarios de primer curso, por lo que es esperable en ellos unas actitudes y competencias acordes con una amplitud de intereses y pensamientos críticos.

$\mathrm{Al}$ tratar de esclarecer posibles diferencias de género entre los factores de personalidad evaluados, el resultado obtenido en Neuroticismo viene a confirmar la investigación de Ruiz et al. (2003), señalando de este modo la importancia notoria entre todos los CIAs de este rasgo. Además, la diferencia encontrada entre hombres y mujeres en Amabilidad permite matizar nueva información que facilita poder describir con mayor precisión a los varones CIA como jóvenes con un carácter más confiado y altruista que las mujeres CIA de su misma edad.

Por otra parte, las diferencias en Impulsividad avalan también parte de la investigación precedente (Adan, 2012; Cortés et al., 2014; Fischer y Smith, 2008; Waldeck y Miller, 1997; Zuckermann y Kuhlman, 2000) remarcando la mayor probabilidad de que los varones CIA realicen comportamientos sin pensar, vivan el momento y no mantengan su atención por períodos prolongados.

Una de las aportaciones más importantes de este trabajo ha sido tratar de determinar el peso o valor que todas estas dimensiones pueden tener a la hora de explicar la conducta de consumo entre los CIAs y las consecuencias psicosociales 
que derivan de la misma. Hasta el momento, la mayor parte de la investigación se ha limitado a identificar dimensiones en las que destacan estos jóvenes, pero sin entrar a valorar el nivel predictivo de cada una de ellas.

Por este motivo, el primero de los objetivos de este trabajo era determinar el valor predictivo de la edad de inicio en el consumo y de los factores de personalidad sobre el consumo semanal de alcohol. Al considerar la muestra total, destaca la edad de inicio como variable con mayor influencia predictiva, mientras que no se obtiene valor explicativo significativo de ninguna de las dimensiones de personalidad evaluadas, a diferencia de lo que sostienen diferentes investigaciones (Hussong, 2003; Ibañez et al., 2010; Martin, 2011; Ruiz et al., 2003). Pero sería un error quedarse con este resultado sin darle importancia a la significación de la variable sexo en esta predicción. No matizar el comentario realizado en función del género enmascararía el peso que sí que tienen algunas de las dimensiones de personalidad. En este caso, cabe destacar la importancia explicativa que entre los varones tiene tanto la edad de inicio como las dimensiones Responsabilidad e Impulsividad. Concretamente, el peso explicativo de ambas supera el obtenido en otras investigaciones (Ibáñez et al., 2008, 2010; Martín, 2011).

Una situación diferente se aprecia entre las mujeres, ya que a pesar de obtener puntuaciones elevadas de las dimensiones de personalidad realmente ninguna de ellas parece tener un peso relevante a la hora de explicar y justificar el consumo de alcohol que realizan.

Estos resultados advierten de la importancia de incidir entre los varones en actividades que trabajen el sentido del deber, la mejora de la gestión del tiempo, el establecimiento de límites o el autocontrol de respuestas impulsivas o agresivas.

Por otra parte, es conveniente continuar indagando en nuevas variables que permitan incrementar la varianza explicada de la conducta de consumo entre las mujeres, facilitando de este modo marcar pautas que garanticen una intervención más óptima.

Además, dada la importancia que ha mostrado tener la edad de inicio en el consumo sobre la cantidad de alcohol ingerida semanalmente entre los CIAs y teniendo en cuenta que este es el patrón de consumo mayoritario en población universitaria en estos momentos (OED, 2012, 2013), se avala la necesidad de continuar potenciando la utilización de todas aquellas medidas que muestren su eficacia dentro de la prevención universal para conseguir el objetivo de retrasar la edad de inicio en el consumo de alcohol. Entre estas medidas se encuentran las referidas a las políticas del consumo de alcohol, ya sean los controles legales, la subida de los precios e impuestos o la reducción del volumen de anuncios (Babor et al., 2003; EMCDDA, 2009; Villalbí y Gual, 2009), así como las que fomentan el desarrollo de las capacidades psicosociales de la persona como las referidas a las habilidades de relación interpersonal, de resistencia a la presión de grupo, la mejora en la resolución de conflictos o el incre- mento de la autoestima (CSAP, 2004). No pueden dejarse de lado la importancia de intervenir al mismo tiempo sobre los agentes sociales que rodean a los jóvenes, especialmente sobre los padres, tratando de promover el cambio de actitudes favorables hacia el alcohol o la mejora de la comunicación familiar (CSAP, 2004; SAMHSA, 2010).

Otro de los objetivos de este trabajo era evaluar la capacidad predictiva de la edad de inicio en el consumo, los factores de personalidad y los gramos de alcohol ingeridos a la semana, sobre el número de consecuencias experimentadas. El resultado obtenido define, como era de esperar, que la edad de inicio en el consumo de alcohol (Benton et al., 2006; Grant et al., 2001; Larimer, Turner, Mallett y Geisner, 2004; Muthen y Muthen, 2000) y la cantidad ingerida de esta sustancia (Neighbours et al., 2007) son las variables con mayor poder explicativo. Pero junto a estas variables, aunque con menor peso, se confirma la importancia explicativa de la Impulsividad y también del Neuroticismo, evidenciándose la tendencia observada en los trabajos revisados (Conrod et al., 2006: Cooper et al., 2000; Fisher y Smith, 2008; Littlefield et al., 2009; Ruiz et al., 2003; Simons et al., 2005; Simons et al., 2004).

Esta realidad señala la necesidad de trabajar, en todo tipo de CIAs, y con objeto de disminuir la incidencia de consecuencias negativas, un conjunto de propuestas sobre el manejo de habilidades de afrontamiento en situaciones de estrés, entre las que podrían incluirse actividades que refuercen el control de impulsos y fomenten el manejo de la ansiedad, de reacciones hostiles y de estados emocionales negativos. Un ejemplo de un programa de intervención que incide directamente en algunas de las variables incluidas en el factor Neuroticismo es el llevado a cabo por Conrod et al. (2006) y Conrod, Castellanos-Ryan y Mackie (2008, 2011) con jóvenes CIA. Éste ha probado su eficacia en repetidas ocasiones en reducir la cantidad de alcohol ingerido, los episodios de CIA y la probabilidad de experimentar problemas derivados del consumo (Conrod et al., 2006; Conrod et al., 2008, 2011; O’Leary-Barrett, Mackie, Castellanos-Ryan, Al-Khudhairy y Conrod, 2010). Si bien, el propio Conrad advierte que a pesar de la evidencia creciente a favor de un enfoque de prevención del consumo de alcohol basado en la personalidad, todavía existen lagunas principalmente en lo que rodea a la generalización de que los efectos a corto plazo sobre la conducta de consumo de alcohol se traduzcan en una reducción del riesgo de experimentar problemas a largo plazo (Conrod et al., 2011).

Este trabajo presenta una serie de limitaciones que es necesario reseñar. En primer lugar, los participantes son estudiantes universitarios, lo que permite cuestionar la generalización de resultados a todos los jóvenes de estas edades. Sería conveniente realizar estudios con muestras de jóvenes universitarios y no universitarios, aunque no puede obviarse la dificultad que supone el poder conseguir acceder a este último colectivo. 
En segundo lugar, las estimaciones de los consumos de alcohol están basados en auto informes y no en medidas objetivas, con los sesgos que esto puede introducir.

Además una limitación que va más allá de este trabajo pero que es importante tener presente es que no se dispone de un consenso internacional en la definición del CIA. Esta heterogeneidad lleva a algunos a estimar el CIA con el número de consumiciones sin especificar período de tiempo en el que se realiza el consumo, ni si las consumiciones son de alcohol de baja o alta graduación, a otros a estimarlo en función de los gramos consumidos considerando un intervalo de ingesta de 2-3 horas, a otros en función del número de veces que se realiza un consumo elevado en los últimos meses, etc. Esta situación lleva a que en muchas ocasiones se esté comparando los resultados obtenidos por sujetos muy heterogéneos, que han sido incluidos bajo una misma denominación. Esto advierte de la necesidad de consensuar la operativización del CIA atendiendo al mayor rigor posible.

Finalmente comentar que dado que las variables incluidas en este estudio sólo permiten dar cuenta de una parte de la conducta de consumo y de las consecuencias derivadas del mismo, sería interesante indagar e incluir otras posibles variables explicativas, como las normas percibidas sobre la ingesta de alcohol por parte del entorno (Neighbors et al., 2007) o los motivos de consumo (Martín, 2011; Mezquita et al., 2010), las cuales han mostrado su eficacia de manera independiente.

\section{Agradecimientos}

Estos resultados forman parte de la investigación en drogodependencias SPI/3462/2010 financiada por el Ministerio de Sanidad y Política Social y dirigido por el Dr. Fernando Cadaveira.

\section{Conflicto de intereses}

No existe ningún conflicto de intereses.

\section{Referencias}

Adan, A. (2012). Impulsividad funcional y disfuncional en jóvenes con consumo intensivo de alcohol. Adicciones, 24, 17-22.

Afitska, N., Plant, M. A., Weir, I., Miller, P. y Plant, M. L. (2008). The relationship between teenage 'binge' drinking, age of first alcohol consumption and intoxication. Journal of Substance Use, 13, 205-218. doi: 10.1080/14659890701740663

Anderson, P. y Baumberg, B. (2006). Alcohol in Europe. London: Institute of Alcohol Studies.

Arata, C. M., Stafford, J. y Tims, M. S. (2003). High school drinking and its consequences. Adolescence, 38, 567-579.

Babor, T., Caetano, R., Casswell, S., Edwards, G., Giesbrecht, N., Graham, K.,... Rossow, I. (2003). Alcohol: no or- dinary commodity: Research and Public policy. Oxford University Press.

Balodis, I. M., Potenza, M. N. y Olmstead, M. C. (2009). Binge drinking in undergraduates: relationships with sex, drinking behaviours, impulsivity, and the perceived effects of alcohol. Behavioral Pharmacology, 20, 518-526. doi: 10.1097/FBP.0b013e328330c779

Benton, S. L., Downey, R. G., Glider, P. S., Benton, S. A., Shin, K., Newton, D. W.,... Price, A. (2006). Predicting negative drinking consequences: Examining descriptive norm perception. Journal of Studies on Alcohol, 67, 399-405.

Blom, G. (1958). Statistical estimates and transformed beta-variables. New York: John Wiley and Sons.

Bonomo, Y. A., Bowes, G., Coffey, C., Carlin, J. B. y Patton, G. C. (2004). Teenage drinking and the onset of alcohol dependence: a cohort study over seven years. Addiction, 99, 1520-1528. doi: 10.1111/j.1360-0443.2004.00846.x

Boyle, G. J., Matthews, G. y Saklofske, D. H. (2008). Personality theories and models: An overview. En G. J. Boyle, G. Matthews y D. H. Saklofske (Eds.), Personality theory and assessment. Personality theories and models (Vol. 1, pp. 1-29). London: SAGE.

Cadaveira, F. (2010). Consecuencias neurocognitivas: estudio de seguimiento en jóvenes españoles que realizan binge drinking. Trabajo presentado en el Seminario Consumo Intensivo de Alcohol en Jóvenes: conocimiento, alternativas y viabilidad. Mayo, Valencia.

Calafat, A. (2007). El abuso de alcohol de los jóvenes en España. Adicciones, 19, 217-224.

Carlson, S. R. y Johnson, S. C. (2012). Impulsivity is not always associated with student drinking: A moderation study of impulsivity and drinking by positive alcohol expectancies. Addictive Behaviors, 37, 556-560. doi: 10.1016/j.addbeh.2011.12.007

Center for Substance Abuse Prevention (2004). SAMHSA Model Programs. Effective Substance Abuse and Mental Health Programs for Every Community. Recuperado de http:/ / modelprograms.samhsa.gov/.

Conrod, P. J., Castellanos, N. y Mackie, C. (2008). Personality-targeted interventions delay the growth of adolescent drinking and binge drinking. Journal of Child Psychology and Psychiatry, 49, 181-190.

Conrod, P. J., Castellanos-Ryan, N. y Mackie, C. (2011). Long-Term Effects of a Personality-Targeted Intervention to Reduce Alcohol Use in Adolescents. Journal of Consulting and Clinical Psychology, 79, 296-306. doi: dx. doi.org/10.1037/a0022997

Conrod, P. J., Stewart, S. H., Comeau, N. y Maclean, A. M. (2006). Efficacy of Cognitive-Behavioral Interventions Targeting Personality Risk Factors for Youth Alcohol Misuse. Journal of Clinical Child and Adolescent Psychology, 35, 550-563. doi: 10.1207/s15374424jccp3504_6

Cooper, M. L., Agocha, V. B. y Sheldon, M. S. (2000). A motivational perspective on risky behaviors: The role of 
personality and affect regulatory processes. Journal of Personality, 68, 1059-1088. doi:10.1111/1467-6494.00126

Cordero, A., Pamos, A. y Seisdedos, N. (2008). NEO PI-R Manual. Adaptación Española. Madrid: TEA Ediciones.

Cortés, M. T. (2010). Debates en drogodependencias: Aspectos sociales y de salud en el botellón: obligados a entenderse. Valencia: Plan Municipal de Drogodependencias. Ayuntamiento de Valencia.

Cortés, M. T. (2012). Determinantes psico-sociales relacionados con el consumo intensivo de alcohol de mujeres. Trabajo presentado en las XIV Jornadas asociación Proyecto hombre. Jóvenes y alcohol: enfocando una realidad, Madrid.

Cortés, M. T., Giménez, J. A., Motos, P. y Cadaveira, F. (2014). The importance of expectations in the relationship between impulsivity and binge drinking among university students. Adicciones, 26, 134-145.

Cortés, M. T., Giménez, J. A., Tomás, I., Espejo, B., Pascual, F., Pedrero, E. y Guardia, J. (2012). Instrumento de Evaluación del Consumo Intensivo de Alcohol. Informe final proyecto de investigación presentado al Plan Nacional sobre Drogas.

Cortés, M. T., Motos, P. y Giménez, J. A. (2013). Adjustment of different ways of measuring alcohol binge drinking in Spanish youngsters. Póster presentado en el Congreso Internacional Global Addiction, Pisa.

Costa, P. T. y McCrae, R. R. (1992). Revised NEO Personality Inventory (NEO-PI-R) and NEO Five-Factor Inventory (NEO-FFI) professional manual. Odessa, FL: Psychological Assessment Resources.

Costa, P. T. y McCrae, R. R. (1999). Inventario de Personalidad NEO Revisado (NEO PIR) e Inventario NEO Reducido de Cinco Factores (NEO-FFI). Manual profesional. Madrid: TEA.

Cyders, M. A., Flory, K., Rainer, S. y Smith, G. T. (2009). The role of personality dispositions to risky behavior in predicting first-year college drinking. Addiction, 104, 193-202. doi:10.1111/j.1360-0443.2008.02434.x

Danielsson, A. K., Wennberg, P., Tengström, A. y Romelsjö, A. (2010). Adolescent alcohol use trajectories: Predictors and subsequent problems. Addictive Behaviors 35, 848-852. doi: 10.1016/j.addbeh.2010.05.001

Dawson, D. A., Goldstein, R. B., Chou, S. P., Ruan, W. J. y Grant, B. F. (2008). Age at first drink and the first incidence of adult-onset DSM-IV alcohol use disorders. Alcoholism Clinical Experimental Research, 32, 2149-2160. doi: 10.1111/j.1530-0277.2008.00806.x

Devos-Comby, L. y Lange, J. E. (2008). Standardized Measures of Alcohol-Related Problems: A Review of Their Use Among College Students. Psychology of Addictive Behaviors, 22, 349-361. doi: 10.1037/0893-164X.22.3.349

DeWit, D. J., Adlaf, E. M., Offord, D. R. y Ogborne, A. C. (2000). Age at first alcohol use: a risk factor for the development of alcohol disorders. American Journal of Psychiatry, 157, 745-750. doi: 10.1176/appi.ajp.157.5.745

European Monitoring Centre for Drugs and Drug Addiction (EMCDDA) (2009). Annual Report 2009. Recuperado de http://www.emcdda.europa.eu/attachements. cfm/att_93236_EN_EMCDDA_AR2009_EN.pdf
Field, M., Schoenmakers, T. y Wiers, R. W. (2008). Cognitive processes in alcohol binges: a review and research agenda. Current Drug Abuse Reviews, 1, 263-279.

Fischer, S. y Smith, G. T. (2008). Binge eating, problem drinking, and pathological gambling: Linking behavior to shared traits and social learning. Personality and individual Differences, 44, 789-800. doi: 10.1016/j.paid.2007.10.008

Flory, K., Lynam, D., Milich, R., Leukefeld, C. y Clayton, R. (2002). The relations among personality, symptoms of alcohol and marijuana abuse, and symptoms of comorbid psychopathology: results from a community sample. Experimental and clinical psychopharmacology, 10, 425-434. doi: 10.1037/1064-1297.10.4.425

Gliksman, L., Adlaf, E. M., Demers, A. y Newton, B. (2003). Heavy Drinking on Canadian Campuses. Canadian Journal of Public Health, 94, 17-21.

Goudriaan, A. E., Grekin, E. R. y Sher, K. J. (2011). Decision making and response inhibition as predictor of heavy alcohol use: a prospective study. Alcoholism: Clinical and Experimental Research, 35, 1-8. doi: 10.1111/j.1530-0277.2011.01437.x

Grant, B. F., Stinson, F. S. y Harford, T. C. (2001). Age at onset of alcohol use and DSM-IV alcohol abuse and dependence: a 12-year follow-up. Journal of Substance Abuse, 13, 493-504. doi: 10.1016/S0899-3289(01)00096-7

Hair, P. y Hampson, S. E. (2006). The role of impulsivity in predicting maladaptative behaviour among female students. Personality and Individual Differences, 40, 943-952. doi: 10.1016/j.paid.2005.10.002

Harford, T. C. (2003). Early onset of alcohol use and health problems: spurious associations and prevention. Addiction, 98, 1483-1484. doi: 10.1046/j.13600443.2003.00555.x

Henges, A. L. y Marczinski, C. A. (2012). Impulsivity and alcohol consumption in young social drinkers. Addictive Behaviors, 37, 217-220.

Hingson, R. W., Assailly, J. P. y Williams, A. F. (2004). Underage drinking: Frequency, consequences, and interventions. Traffic Injury Prevention, 5, 228-236. doi: $10.1080 / 15389580490465256$

Hingson, R. W., Heeren, T. y Winter M. R. (2006). Age at drinking onset and alcohol dependence: age at onset, duration, and severity. Archives of pediatrics $\mathcal{E}$ adolescent medicine, 160, 739-746. doi: 10.1542/peds.2006-0223

Hingson, R. W. y Zha, W. (2009). Age of drinking onset, alcohol use disorders, frequent heavy drinking, and unintentionally injuring oneself and others after drinking. Pediatrics, 123, 1477-1484. doi: 10.1542/peds.2008-2176

Hingson, R., Zha, W. y Weitzman, E. R. (2009). Magnitude of and trends in alcohol-related mortality and morbidity among US college students ages 18-24. Journal of Study on Alcohol and Drugs, supl. 16, 12-20.

Hopwood, C. J., Morey, L. C., Skodol, A. E., Stout, R. L., Yen, S., Ansell, E. B.,... McGlashan, T. H. (2007). Five-factor model of personality traits associated with al- 
cohol-related diagnoses in a clinical sample. Journal of Studies on Alcohol and Drugs, 68, 455-460.

Hussong, A. M. (2003). Social Influences in Motivated Drinking Among College Students. Psychology of Addictive Behaviors, 17, 142-150. doi: 10.1037/0893-164X.17.2.142

Ibáñez, M. I., Moya, J., Villa, H., Mezquita, L., Ruipérez, M. A. y Ortet, G. (2010). Basic personality dimensions and alcohol consumption in Young adults. Personality and Individual Differences, 48, 171-176. doi: 10.1016/j. paid.2009.09.017

Ibáñez, M. I., Ruipérez, M. A., Villa, H., Moya, J. y Ortet, G. (2008). Personality and alcohol use. En G. J. Boyle, G. Matthews y D. H. Saklofske (Eds.), Personality theory and assessment. Personality theories and models (Vol. 1, pp. 677-697). London: SAGE.

Jenkins, M. B., Agrawal, A., Lynskey, M. T., Nelson, E. C., Madden, P. A., Bucholz, K. K. y Heath, A. C. (2011). Correlates of Alcohol Abuse/Dependence in Early-Onset Alcohol-Using Women. The American Journal on Addictions, 20, 429-434. doi: 10.1111/j.15210391.2011.00151.x.

Kahler, C. W., Strong, D. R. y Read, J. P. (2005). Toward efficient and comprehensive measurement of the alcohol problems continuum in college students: The Brief Young Adult Alcohol Consequences Questionnaire. Alcoholism: Clinical and Experimental Research, 29, 11801189.doi:10.1097/01.ALC.0000171940.95813.A5

Kubicka, L., Matjcek, Z., Dytrych, Z. y Roth, Z. (2001). IQ and personality traits assessed in childhood as predictors of drinking and smoking behaviour in middle-aged adults: a 24-year follow-up study. Addiction, 96, 16151628. doi: 10.1111/j.1467-6494.1996.tb00816.x

Larimer, M. E., Turner, A. P., Mallett, K. A. y Geisner, I. M. (2004). Predicting drinking behavior and alcohol-related problems among fraternity and sorority members: Examining the role of descriptive and injunctive norms. Psychology of Addictive Behaviors, 18, 203-212. doi: 10.1037/0893-164X.18.3.203

Littlefield, A. K., Sher, K. J. y Wood, P. K. (2009). Is the "maturing out" of problematic alcohol involvement related to personality change? Journal of Abnormal Psychology, 118, 360-374. doi: 10.1037/a0015125

Littlefield, A. K., Sher, K. J. y Wood, P. K. (2010). A personality-based description of maturing out of alcohol problems: Extension with a five-factor model and robustness to modeling challenges. Addictive Behaviors, 35, 948-954. doi: 10.1016/j.addbeh.2010.06.008

Livingston, M., Laslett, A. M. y Dietze, P. (2008). Individual and community correlates of young people's high-risk drinking in Victoria, Australia. Drug Alcohol Depend, 98, 241-248. doi: 10.1016/j.drugalcdep.2008.06.002

Lo, C. C. (1996). Are women heavier drinkers than we thought they were? Journal of Studies on Alcohol, 57, 531-535.
Locke, T. F. y Newcomb, M. D. (2001). Alcohol involvement and dysphoria: A longitudinal examination of gender differences from late adolescence to adulthood. Psychology of Addictive Behaviors, 15, 227-236. doi: 10.1037/0893164X.15.3.227

MacKillop, J., Mattson, R. E., Anderson, E. J., Castelda, B. A. y Donovick, P. J. (2007). Multidimensional assessment of impulsivity in undergraduate hazardous drinkers and controls. Journal of Studies on Alcohol and Drugs, 68, 785-788.

Magid, V., MacLean, M. G. y Colder, C. R. (2007). Differentiating between sensation seeking and impulsivity through their mediated relations with alcohol use and problems. Addictive Behaviors, 32, 2046-2061. doi: 10.1016/j.addbeh.2007.01.015

March, J. C., Prieto, M. A., Danet, A., Escudero, M., López, M. y Luque, N. (2010). El consumo de alcohol en los adolescentes: una aproximación cualitativa desde los docentes. Trastornos Adictivos, 12, 65-71. doi: 10.1016/ S1575-0973(10)70013-1

Mallett, K. A., Marzell, M., Varvi-Weld, L., Turrisi, R., Guttman, K. y Abar, C. (2011). One-Time or Repeat Offenders? An Examination of the Patterns of Alcohol-Related Consequences Experienced by College Students Across the Freshman Year. Addictive Behaviors, 36, 508-511. doi: 10.1016/j.addbeh.2010.12.022

Martens, M. P., Ferrier, A. G., Sheehy, M. J., Corbett, K., Anderson, D. A. y Simmons, A. (2005). Development of the protective behavioral strategies survey. Journal of Studies on Alcohol, 66, 698-705.

Martin, J. L. (2011). Examination of the interaction of drinking motives and personality on alcohol use and alcohol-related problems among college students. (Order No. 3464655, State University of New York at Albany). ProQuest Dissertations and Theses, 157. Recuperado de http:/ / search.proquest.com/ docview/886751150?accountid=14777. (886751150).

Martin, C. S., Lynch, K. G., Pollock, N. K. y Clark, D. B. (2000). Gender differences and similarities in the personality correlates of adolescent alcohol problems. Psychology of Addictive Behaviors, 14, 121-133. doi: 10.1037/0893-164X.14.2.121

Meda, S. A., Stevens, M. C., Potenza, M. N., Pittman, B., Gueorguieva, R., Andrews, M. M., ... Pearlson, G. D. (2009). Investigating the behavioral and self-report constructs of impulsivity domains using principal component analysis. Behavioral Pharmacology, 20, 390-399. doi: 10.1097/FBP.0b013e32833113a3

Mestre, H., Viñas, M., Dutil, V. y Moya, J. (2004). Personalidad y consumo de alcohol en adolescentes. Trabajo presentado en las Jornadas de fomento de la investigación de la Universitat Jaume I, Castellón de la Plana.

Mezquita, L., Stewart, H. S. y Ruipérez, M.A. (2010). Big-five personality domains predict internal drinking motives in Young adults. Personality and Individual Differences, 49, 240-245. doi: 10.1016/j.paid.2010.03.043 
Ministerio de Sanidad y Consumo (2008). Prevención de los problemas derivados del alcohol. $1^{a}$ Conferencia de prevención y promoción de la salud en la práctica clínica en España. Madrid: Ministerio de Sanidad y Consumo.

Motos, P. (2013). Determinantes del consumo intensivo de alcohol en jóvenes universitarios (Tesis Doctoral). Valencia: Universitat de València.

Muthen, B. y Muthen, L. (2000). The development of heavy drinking and alcohol-related problems from ages 18 to 37 in a U.S. national sample. Journal of Studies on Alcohol, 61, 290-300.

National Institute on Alcohol Abuse and Alcoholism (2004). Council approves definition of binge drinking. NIAAA Newsletter, 3.

Neighbors, C., Lee, C. M., Lewis, M. A., Fossos, N. y Larimer, M. E. (2007). Are Social Norms the Best Predictor of Outcomes Among Heavy-Drinking College Students? Journal on Studies on Alcohol and Drugs, 68, 556-565.

Neighbors, C., Walker, D. D. y Larimer, M. E. (2003). Expectancies and evaluations of alcohol effects among college students: Self-determination as a moderator. Journal of Studies on Alcohol, 64, 292-300.

Observatorio Español sobre Drogas del Plan Nacional Sobre Drogas (2012). Encuesta Domiciliaria sobre Alcohol y Drogas en España, EDADES 2011-2012. Madrid: Ministerio de Sanidad, Servicios Sociales e Igualdad. Recuperado de http:/ / www.pnsd.msssi.gob.es/Categoria2/observa/pdf/EDADES2011.pdf

Observatorio Español sobre Drogas del Plan Nacional Sobre Drogas (2013). Encuesta Estatal sobre el Uso de Drogas en Enseñanzas Secundarias, ESTUDES 2012-2013, España. Madrid: Ministerio de Sanidad, Servicios Sociales e Igualdad. Recuperado dehttp://www.pnsd.msssi.gob.es/Categoria2/ observa/pdf/PresentESTUDES2012_2013.pdf

O'Leary-Barrett, M., Mackie, C. J., Castellanos-Ryan, N., Al-Khudhairy, N. y Conrod, P. J. (2010). Personality-targeted interventions delay uptake of drinking and decrease risk of alcohol-related problems when delivered by teachers. Journal of the American Academy of Child E Adolescent Psychiatry, 49, 954-963. doi: 10.1016/j.jaac.2010.04.011

O'Malley, P. M. y Johnston, LL. D. (2002). Epidemiology of Alcohol and Other Drug Use among American College Students. Journal of Studies on Alcohol, 14, 23- 39.

Oquendo, P. A., Baca-García, E., Graver, R., Morales, M., Montalbán, V. y Mann, J. J. (2001). Spanish Adaptation of Barrat Impulsiveness Scale (BIS). European Journal of Psychiatry, 15, 147-155.

Parada, M., Corral, M., Caamaño-Isorna, F., Mota, N., Crego, A., Rodríguez, S. y Cadaveira, F. (2011). Definición del concepto de consumo intensivo de alcohol adolescente (binge drinking). Adicciones, 23, 53-63.

Park, C. L. (2004). Positive and negative consequences of alcohol consumption in college students. Addictive Behaviors, 29, 311-321. doi: 10.1016/j.addbeh.2003.08.006
Patton, J. H., Stanford, M. S. y Barratt, E. S. (1995). Factor structure of the Barratt Impulsiveness Scale. Journal of Clinical Psychology, 51, 768-774. doi: 10.1002/10974679(199511)51: 6<768::AID-JCLP2270510607>3.0.CO;2-1

Pilatti, A., Caneto, F., Garimaldi, J. A., Del Valle, B. y Pautassi, R. M. (2013). Contribution of Time of Drinking Onset and Family History of Alcohol Problems in Alcohol and Drug Use Behaviors in Argentinean College Students. Alcohol and Alcoholism, 49, 128-137. doi: 10.1093/ alcalc/agt176

Pitkänen, T., Lyyra, A. L. y Pulkkinen, L. (2005). Age of onset of drinking and the use of alcohol in adulthood: a follow-up study from age 8-42 for females and males. Addiction, 100, 652-61. doi:10.1111/j.1360-0443.2005.01053.x

Prado, V., Crespo, J. M., Brenlla, J. y Páramo, M. (2007). Relación entre consumo de alcohol y rasgos patológicos de personalidad en una muestra de alumnos universitarios. Trastornos Adictivos, 9, 126-131.

Quilty, L. C. y Oakman, J. M. (2004). The assessment of behavioral activation: The relationship between impulsivity and behavioral activation. Personality and Individual $\mathrm{Di}$ fferences, 37, 429-442. doi: http://dx.doi.org/10.1016/j. paid.2003.09.014

Ray, A. E., Turrisi, R., Abar, B. y Peters, K. E. (2009). Social-cognitive correlates of protective drinking behaviors and alcohol-related consequences in college students. Addictive Behaviors, 34, 911-917. doi: 10.1016/j.addbeh.2009.05.016

Read, J. P. y O'Connor, R. M. (2006). High-and low-dose expectancies as mediators of personality dimensions and alcohol involvement. Journal of Studies on Alcohol and Drugs, 67, 204-214.

Rodríguez-Martos, A., Gual, A. y Llopis, J. J. (1999). La unidad de bebida estándar como registro simplificado del consumo de bebidas alcohólicas y su determinación en España. Medicina Clinica, 112, 446-450.

Rossow, I. y Kuntsche, E. (2013). Early onset of drinking and risk of heavy drinking in young adulthood-a 13year prospective study. Alcoholism: Clinical and Experimental Research, 37, E297-E304. doi: 10.1111/j.15300277.2012.01924.x

Ruipérez, M. A., Ibáñez, M. I., Villa, H. y Ortet, G. (2006). Factores biopsicosociales en el consumo de alcohol. En L.A. Oblitas (Ed.), Atlas de psicología de la salud. Bogotá: PSICOM editores.

Ruiz, M. A., Pincus, A. L. y Dickinson, K. A. (2003). NEO PI-R Predictors of Alcohol Use and Alcohol-Related Problems. Journal of Personality Assessment, 81, 226-236. doi: 10.1207/S15327752JPA8103_05

Sanz, J., Silva, F. y Avia, M. D. (1999). La evaluación de Personalidad desde el modelo de los "Cinco Grades": El Inventario de Cinco- Factores NEO (NEO-FFI) de Costa y McCrae. En F. Silva (Ed.), Avances en Evaluación Psicológica (pp. 171-234). Valencia: Promolibro. 
Schuckit, M. A., Tipp, J. E., Bergman, M. y Reich,W. (1997). Comparison of induced and independent major depressive disorders in 2,945 alcoholics. American Journal of Psychiatry, 154, 948-957.

Schutte, K. K., Hearst, J. y Moos, R. H. (1997). Gender differences in the relations between depressive symptoms and drinking behavior among problem drinkers: a three-wave study. Journal of consulting and clinical psychology, 65, 392.

Sher, K. J., Grekin, E. R. y Williams, N. A. (2005). The development of alcohol use disorders. Annual Review of Clinical Psychology, 1, 493-523. doi: 10.1146/annurev. clinpsy.1.102803.144107

Sher, K. J., Trull, T. J., Bartholow, B. D. y Veith, A. (1999). Personality and alcoholism: Issues, methods, and etiological processes. En K. E. Leonard y H. T. Blane (Eds.), Psychological theories of drinking and alcoholism (2nd ed., pp. 54-105). New York: Guilford Press.

Shield, K. D., Gmel, G., Patra, J. y Rehm, J. (2012). Global burden of injuries attributable to alcohol consumption in 2004: a novel way of calculating the burden of injuries attributable to alcohol consumption. Population Health Metrics, 9, 1-14.

Simons, J. S., Carey, K. B. y Gaher, R. M. (2004). Lability and impulsivity synergistically increase risk for alcohol-related problems. The American Journal of Drugs and Alcohol Abuse, 30, 685-694. doi:10.1081/ADA-200032338

Simons, J. S., Gaher, R. M., Correia, C. J., Hansen, C. L. y Christopher, M. S. (2005). An affective-motivational model of marijuana and alcohol problems among college students. Psychology of Addictive Behaviors, 19, 326-334. doi: 10.1037/0893-164X.19.3.326

Stanford, M. S., Mathias, C. W., Dougherty, D. M., Lake, S. L., Anderson, N. E. y Patton, J. H. (2009). Fifty years of the Barratt impulsiveness scale: An update and review. Personality and Individual Differences, 47, 385-395. doi: 10.1016/j.paid.2009.04.008

Stewart, S. H., Loughlin, H. L. y Rhyno, E. (2001). Internal drinking motives mediate personality domain-drinking relations in young adults. Personality and Individual Differences, 30, 271-286. doi: 10.1016/S0191-8869(00)00044-1

Substance Abuse and Mental Health Services Administration (2010). Town Hall Meeting. Mobilizing Communities to Prevent and Reduce Underage Alcohol Use. Recuperado de http://store.samhsa.gov

Villalbí, J. R. y Gual, A. (2009). Las políticas preventivas del consumo de sustancias adictivas y la responsabilidad de los profesionales. Adicciones, 21, 5-8.

Waldeck, T. L. y Miller, L. S. (1997). Gender and impulsivity differences in licit substance use. Journal of Substance Abuse, 9, 269-275.

Warner, L. A. y White, H. R. (2003). Longitudinal effects of age at onset and first drinking situations on problem drinking. Substance Use and Misuse, 38, 1983-2016. doi: 10.1081/JA-120025123
Warner, L. A., White, H. R. y Johnson, V. L. (2007). Alcohol initiation experiences and family history of alcoholism as predictors of problem drinking trajectories. Journal of Studies on Alcohol, 68, 56-65.

Wechsler, H., Dowdall, G. W., Davenport, A. y Castillo, S. (1995). Correlates of student binge drinking. American Journal of Public Health, 85, 921-926. doi: 10.2105/ AJPH.85.7.921

Wechsler, H. y Nelson, T. F. (2010). Will increasing alcohol availability by lowering the minimum legal drinking age decrease drinking and related consequences among youths? American Journal of Public Health, 100, 986-992. doi: 10.2105/AJPH.2009.178004

White, A. M., Kraus, C. L. y Swartwelder, H. S. (2006). Many College Freshman Drink at Levels Far Beyond the Binge Threshold. Alcoholism: Clinical and Experimental Research, 30, 1006-1010. doi: 10.1111/j.1530-0277.2006.00122.x

White, H. R., Marmorstein, N. R., Crews, F. T., Bates, M. E., Mun, E. Y. y Loeber, R. (2011). Associations between heavy episodic drinking and changes in impulsive behavior among adolescent boys. Alcoholism: Clinical and Experimental Research, 35, 295-303.

Whiteside, S. P. y Lynam, D. R. (2001). The five factor model and impulsivity: Using a structural model of personality to understand impulsivity. Personality and Individual Differences, 30, 669-689. doi: 10.1016/S01918869(00) 00064-7

Woicik, P. A., Stewart, S. H., Pihl, R. O. y Conrod, P. J. (2009). The substance use risk profile scale: A scale measuring traits linked to reinforcement-specific substance use profiles. Addictive Behaviors, 34, 1042-1055. doi: 10.1016/j.addbeh.2009.07.001

World Health Organization (2007). WHO Expert Committee on Problems Related to Alcohol Consumption. Second report. Geneva (WHO Technical Report Series, No. 944).

Zuckerman, M. y Kuhlman, D. M. (2000). Personality and risk-taking: common biosocial factors. Journal of Personality, 68, 999-1029. 